\title{
संगीत कला में कंठ साधना का महत्व
}

\section{डा॰ हरजस कौर}

एसोसिऐट प्रोफैसर, संगीत विभाग, सरकारी कालेज, रोपड़

संगीत कला ईश्वर प्रदत्त प्रतिभा है लेकिन मनुष्य में परिमित है संगीत कंठ गायन और वादित ध्वनियों के संगम से आनंदाभास करवाता है, जो मानव जीवन का अभिन्न अंग है। आनंद का माघ्यम जितना सुक्ष्म होगा आनंद उतना अधिक और उच्च स्तरीय होगा। कंठ ध्वनियां भी संगीत कला का सूक्ष्म आनंद माध्यम है, जो तन और मन की कड़ी साधना से अपना स्वरूप आवाज के तौर पे निर्मित करती हैं। इसमें मानव कंठ से विभिन्न विधियों के द्वारा मनवांछित आवाज उत्पन्न की जाती है जो कंठ साधना भाव अभ्यास से संगीत कौशल में परिवर्तित हो जाती है।

मानव शरीर में ध्वनि उत्पति स्थान को स्वरयंत्र का नाम दिया गया है जो मस्तिष्क, कान, ध्वनियंत्र, कर्णयंत्र और फेफडों की सहायता से कार्यशील होता है। यह प्रत्येक व्यक्ति के शरीर में भाग की तरह विद्यमान तो रहते है लेकिन वाणी की गुणवत्ता इसे विशिष्ट गुण के रूप में अभिव्यक्त करती है। यह साधारण रूप से तो केवल बोल-चाल तक ही सीमित रहती है लेकिन इन्ही स्वरों को साधना के द्वारा प्रभावशाली बना कर संगीत गुण में विकसित किया जा सकता है। इस लिए व्यक्ति विशेष में यह प्राकृतिक गुण के रूप में पाया जाता है लेकिन स्वर साधना के द्वारा इसे अर्जित भी किया जा सकता है।

प्राकृतिक गुण के तौर पर कंठ स्थान को एक कोमलयंत्र की ध्वनि और प्रतिध्वनि पैदा करने वाला स्थान माना जाता है। कंठ के स्नायुओं द्वारा ध्वनि का निर्माण होता है। ध्वनि के लिए हमारी श्वासनलिका, फेफड़े तथा गले की खोखली सहायक होती है। अतः इन सबका कार्य सुचारू ढंग से चले इसके लिए शरीर के इन भागों का स्वस्थ होना आवश्यक है ताकि वह कंठ की आवाज पर नियंत्रण बना सकें जिससे कार्टीलेज स्वास नली ऊपर के भाग में स्थित होते हैं जो ऊँची आवाज़ के अनुसार सिकुड़ते और फैलते हैं प्राय: यह विशेषता प्राकृतिक गुण की वजह से होती है। प्राकृतिक विशेषता के अनुसार यह फिर उच्च, मध्यम और धीमी में पाई जाती है जो कभी कभी व्यक्ति के स्वभाव पर भी आधारित होती हैं कि किसी का कंठ सुरीला है और किसी का कठोर बनता हैं। जैसे औरतों और पुरूषों की आवाज 
में अन्तर होता है। परन्तु संगीतकारों की दृष्टि में स्वर साधना के द्वारा सुरीले कंठ को और मधुर और आनंददायक बनाया जा सकता है स्वर की साधना से अर्जित किया जा सकता है इस लिए उन्होने कंठ स्थान को 'सरस्वती ${ }^{1}$ का स्थान दिया। जिसके लिए कंठ ध्वनि को आदरमय ढंग से आदेशनुसार साधना चाहिए। गायन के समय कंठ, गर्दन, मुंह तथा होठों के स्नायु भी स्वतन्त्र रखने चाहिए। अच्छी आवाज़ के लिए गले को सुयोग्य आकार में साधकर कंठ स्थान से आवाज़ निकालनी चाहिए। यह सभी लगन और मेहनत के बल पर ही साधा जा सकता है। प्रत्येक अच्छे गायक का यही मानना है कि संगीत सीखने के लिए कंठ ध्वनि को साधना पड़ता है। एक ही राग को बार बार गाओ ताकि उसका स्वरोच्चार, सदाचार शुद्व हो जाये और आवाज़ सही स्थान पर बैठ जाये तब अगले रागों का अभ्यास आसान हो जाता है। कंट साधना के समय मंद्र सप्तक के स्वरों का बार-बार उच्चारण किया जाता है। मध्यम सप्तक के षड़ज स्वर पर कम से कम 10 बार आवाज़ लगाई जाती है। इस तरह मंद्र सप्तक के स्वरों पर आरोहावरोह के रूप में आवाज़ पूर्ण स्वास से लगाई जाती है यहां गाने वाला स्वंय नियंत्रण करता है कि जहां कहीं बेसुरापन है उसे ठीक करना, जहां आवाज़ कांपती हो उसे ठीक करना और उच्चारण पद्वति पर ध्यानपूर्वक काम करना। सप्तक कंठ साधना में अलंकारों का अम्यास भी आ जाता हे। कंठ साधना एक ऐसा अभ्यास है जो पूर्ण श्वास पर निर्भर है। पूर्ण श्वास में गाकर ही आवाज़ की हरकत को सही स्थान पर लाया जा सकता है। कंठ संगीत में छह प्रकार की साधना की जाती है जिसमें श्वसन, माधुर्य, ध्वनि की गहराई, सुरीलापन और यहां तक कि स्वर मर्यादा और शिक्षक का मार्ग दर्शन भी आता है। जैसे इस संदर्भ में यह धारना है कि गुरू की उपस्थिति में ऊँ शब्द के उच्चारण से कंठ सुरीला होता है जैसे

'When you sing or hum, you use your voice as a musical instrument. Most people sing without any special training they sing with their natural voices and can bring out pleasant sounds without conscious efforts. Others may have good, resonant voices, but they are not able to use them so that they sound well. In either case training will probably improve the natural voices. The rich, beautiful voice may become more beautiful. The food voice may become better. A good voice teacher is important. 2

ऐसा भी नहीं है कि किसी भी कंठ को साधा जा सकता है। प्राकृतिक तौर पर मिली आवाज़ कंठ साधना का आधार है। इसमें गुरू एवम् शिक्षक की सक्रिय भूमिका 
महत्वपूर्ण है। वह अपने भव्य जादुमई ज्ञान से कंठ ध्वनि को स्वरदायक बना सकता है और बेसुरेपन को दूर कर सकता है। उसका अपना अनुभव इस कार्य मे चमत्कार कर सकता है।

प्राचीन काल में कंठ संगीत प्राकृतिक गुण की वजह से खोजा गया। उस काल में धार्मिक मंत्र-उच्चारण के दौरान इसकी विधियां प्रयोग होती थी जो कि श्रोतागण को आनंदविभोर कर देती थी। वीणा मानकरण इसे स्पष्ट करते लिखते है, 'प्राचीन समय में स्वर साधना का विशेष नियम नहीं था, अपितु ऋग्वेद की ऋचाएं और साम गायन के मंत्रों, श्लोकों का सही उच्चारण नियमित रूप से करना ही स्वर साधना थी। उस समय संगीत गायन संस्कृत काव्य में था। धीरे धीरे जब ध्रुवपद, धमार शैलियां प्रचलित हुई तो इससे अच्छी आवाज़ बनी क्योंकि ये गायन आरम्भ में ही नारायण हरि और तुम ही अनन्त हरि आदि शब्दों का उच्चारण करते थे और यह शब्द ही आवाज़ का एक अभ्यास थे। आज इन शब्दों में नोम तोम आदि का रूप ग्रहण कर लिया है। इसी काल में सामवेद ग्रन्थ जो सम्पूर्ण तौर पर संगीतमय रचना है उसमें अंकित मत्रों का गाने का प्रचलन तीन स्वरों उदात्त मन्दर, अनुदात्त तार और स्वरित के तौर पर हुआ। बाद में उदात्त मन्दर, अनुदात्त तार और स्वरित मध्य में इनका उच्चारण आरम्भ हुआ जो बाद में सामने आया। वैदिक संगीत में कंठित स्वरों के तौर पे सामने आया। वैदिक संगीत में कंठित स्वरों के तौर वे सात स्वर प्रयोग किये जाते थे जैसे कृष्ण-मध्यम, प्रथमा-गांधार, द्वितीया-रिषभ, तृतीया-षड़ज, चतुर्थ-निषाद, मन्दर-धौवत, अतिस्वर-पंचम। वैदिक मंत्रों का सही और शुद्ध उच्चारण, नीचे-ऊपर ठहराव आदि को ध्यान में रखते हुए कई गायन शैलियां और लय के विभिन्न प्रकार उत्पन्न हुए। चन्द्र कान्ता खोसला के शब्दों में एक स्वर वाला 'आर्चीका', दो स्वरों वाली 'गीतिका' में परवर्तित हुआ और बाद में तीन स्वरों पर आधरित 'सामिका' का सृजन हुआ। यह गति यहीं पर समाप्त नहीं हुई। चार स्वरों का स्वरान्तर पाँच स्वरों का 'औढ़व' और छ: स्वरों वाला षाड़व, इस तरह एक बाद दूसरा नियम आता है। शिखर पर पहुंच कर सम्पूर्ण सात स्वरों का सप्तक संगीत को प्राप्त हुआ। प्रो. तारा सिंह के मत अनुसार मतंग कृत 'बृहदेशी' और 'नारदीय' शिक्षा के अध्ययन से सिद्व होता है कि वैदिक काल में चार प्रकार की गायन शैलियां रिक, गाथा, सामगान, स्वरान्तर प्रचार में थे। रिक: केवल एक ही स्वर में बंद उच्चारण की शैली को रिक कहा जाता था। सामगान तीन स्वर समूह 
में जो श्लोक और मंत्रा पढ़े जाते थे उस ढंग को 'सामगान' कहा जाता था। इन सब विभिन्न गायन शैलियों से गायकों के घरानों का जन्म हआ जो मंत्र की अपनी-अपनी शैली में गायक करते थे। कनतुव वंश जो ऋषि साम के पहले 50 सूक्त और आठवें मंण्डल का गायन करते थे। दूसरे मण्डल के गायन करने वाने ग्रतसमंद वंश के ट्टि थे। तीसरा मण्डल विश्वामित्रा, चौथा वामदेव और पांचवा अतृय, छठा वृहस्पति और सातवां विष्शिट वंश के घराने के गायको द्वारा गाया जाता था। नवें मण्डल को सारे ही घराने गाते थे।

मध्यकानील समय तक कंठ संगीत का यह वंश एवम् घराना परम्परा राज दरबारों का श्रृगांर बनी जिससे कंठ संगीत में कई-नई गायन शैलियों का प्रयोग शुरू हुआ। जैसे कव्वाली, गजल, ख्याल, ठुमरी और समूह गान आदि। ${ }^{6}$ कहा जाता है कि 'गोपाल और अमीर खुसरो दिल्ली दरबार में आया तो उस श्रेणी का गायन किया जिसे गीत कहते हैं। इस योग्य संगीतकार ने शक्तिशाली और मधुर कंठ की शैली के सौन्दर्य में बढ़ावा किया। इस काल में कंट संगीत विभिन्न धर्मों के अन्तरगत प्रचार में आया।

घराना परम्परा में ख्याल गायकी ज्यादा प्रचलन में आई। पहले ग्वालियर घराने में प्रचलित हुई कंठ संगीत की यह पद्वति आकार में स्पष्ट आलाप की थी। उसके बाद इसे ख्याल गायकी के पटियाला, आगरा किराना, जयपुर घराने बने और इन सबने कंठ साधना के भिन्न-भिन्न ढंग अपनाये। ख्याल गीत संक्षिप्त होता है। इस गायकी में गायक की पूर्ण स्वतन्त्रता रहती है। अतः राग की गम्भीरता व शुद्धता में कमी आनी स्वाभाविक है। गीत गाने से पूर्व पहले थोड़ा सा आलाप किया जाता है। इसमें बराबर की तान, दोगुनी और चौगुनी तानें ली जाती है। इसके बाद में एक ख्याल लोक संगीत परम्परा कंठ संगीत में सामने आई जो ध्रुपद शैली से आगे का विकास है। कंठ संगीत की यह परम्पराएं मूल रूप में प्राकृतिक गुण विशेष रूप में विकसित हुई। लेकिन वर्तमान युग में कंठ संगीत को प्रतिभा के तौर पर प्रतिपादित किया जाता है जो कि कला कौशल के साथ-साथ व्यवसायिकरण में सहायक होता है। कंट संगीत शिक्षण पद्वति भी संस्थागत हो चुकी है। कंट संगीत का परम्परागत दर्शन अब आधुनिक परिपेक्ष में पाठयक्रम विशेष बन कर रह गया है। डा गुरनाम सिंह भी इस बारे में ऐसा ही मत पेश करते है, 'वर्तमान काल में भी परम्परागत सिखलाई के अधीन कंट साधना पर विशेष ध्यान दिया जाता है। यूरोप में स्वर 
साधना महत्वपूर्ण और स्वतंत्रा विषय है। यूरोप में कई ऐसे स्कूल हैं, जहां कंठ साधे जाते हैं। भारतीय परम्परा के अनुसार कंठ साधना विधि पूर्वक लागू होने वाला अनुशासन है।

आरम्भ में प्राकृतिक गुण के रूप में ही बोद्धिकता के स्तर पर संगीत कला का रूप बना और सांगीतक संभावना, उपयोगी बनी परन्तु अब कंठ संगीत की पृष्ठभूमि ही बदल गई। संगीत का आचरण उसका माधुर्य, उसका भाव, उसकी अनुभूति एवमं अभिव्यक्ति अब संस्थागत हो चुकी है।

संस्थागत प्रणाली में अधिक से अध्कि विद्यार्थियों को कंट संगीत की शिक्षा एक साथ दी जाती है। इस सम्बन्ध में सैमीनार, गोष्ठियां इत्यादि भी होती हैं। विद्धजन इस संदर्भ में खोज कार्य कर रहे हैं कि कैसे कंठ संगीत शिक्षण को और प्रतिभाशाली बनाया जाए। शिक्षण स्थलों पर शिष्य चुनाव की कसौटी भी द्रष्टव्य है ताकि प्रतिभा खोज में आसानी हो। इस प्रणाली में शिक्षक ने एक निश्चित पाठयक्रम के अन्तर्गत रियाज़ पक्ष को गौण कर दिया गया है। जिसमें प्रस्तुतिकरण पर जोर बढ़ गया है। यह पद्वति का प्रभाव व्याप्त तो हो गया है लेकिन यह खर्चीली और लम्बी पद्वति है। संगीतकारों का मानना है कि कंठ संगीत शिक्षण में गुरू-शिष्य परम्परागत पद्वति का प्रभाव पैना और गहरा है।

आज ग्लोबलाईजेशन के युग में साज़ और अन्य आवाजें इतनी प्रभावशाली हो गई है कि केवल कंठ संगीत की तरफ से लोगों का घ्यान हट सा गया है। इन्टर्नेट और इलैक्ट्रोनिक मीडीया के दुष्प्रभाव पर सब इतने प्रभावित हैं कि संगीत अपना परम्परागत स्वरूप खो रहा है। कंठ साधना की कमी और विद्यार्थियों की अभिरूचि के कारण इसके शिक्षण और गुणवत्ता दोनों में कमी आई है। कहीं-कहीं लोक संगीत में यह परम्परागत स्वरूप के सहित बचा हे। आज कंठ संगीत योग्यता की खोज दुर्लभ होती जा रही है। ऐसी दुर्गम परिस्थितियों में हमें कंठ संगीत के परम्परागत स्वरूप को कायम रखना पढ़ेगा तभी कंठ ध्वनियों एवम् कंठ संगीत को अनुपम कला के तौर पर कायम रखने का प्रयत्न सफल होगा।

\section{सहायक स्रोत}

1 पं दामोदर, संगीत दर्पण, अनुवादक भटट, पं विश्वम्भरनाथ संगीत कार्यालय, हाथरस, संस्करण 1950 पृष्ठ-11

2 The new book of knowledge, Grolier Incorporated Danbury, Conn, 1980 
3 वीणा मानकरण, संगीत सार, राज पब्लिशर्ज रजि जालन्धर, पृष्ठ 89

4 चन्द्र कान्ता खेसला, भारतीय संगीत दा इतिहास, पृष्ठ 53

5 प्रो तारा सिंह, सुरजीत कौर, वादन कला, पृष्ठ 233

6 अरूण कुमार सेन भारतीय तालों का शासत्रीय विवेचन मध्य प्रदेश, हिन्दी ग्रन्थ अकादमी, भोपाल, 1973 पृष्ठ 26

7 Capt. Willard, A Treasise on the Music of Hindustan, N-A Calcutta, 1834, Page 160

8 डा गुरनाम सिंह, संगीत निंबधावली, पब्लीकेशन व्योरा, पंजाबी युनीवरसिटी पटियाला, 1991, पृष्ठ 16 